\title{
Storage of electrons in shallow donor excited states of GaP:Te
}

\author{
S. D. Ganichev,* W. Raab, E. Zepezauer, and W. Prettl \\ Institut für Experimentelle und Angewandte Physik, Universität Regensburg, 93040 Regensburg, Germany \\ I. N. Yassievich \\ A. F. Ioffe Physicotechnical Institute of the Russian Academy of the Sciences, St. Petersburg, 194021, Russia
}

(Received 3 July 1996)

\begin{abstract}
Tellurium donors in GaP have been ionized by phonon-assisted tunneling in the electric field of pulsed far-infrared laser radiation. In response to the laser pulse a photoconductive signal has been detected with a fast component that follows in time the laser pulse and a slow component that rises after the irradiation has ceased and finally exponentially decays with a strongly temperature-dependent time constant varying from several microseconds to milliseconds. It is shown that this temporal structure of the signal is due to a storage of carriers in the valley-orbit split $1 s(E)$ shallow donor state. Observation of far-infrared to mid-infrared upconversion demonstrates that the final step of cascade recombination is achieved by radiative transitions. [S0163-1829(97)03103-2]
\end{abstract}

Metastable states like $D X$ centers and $E L 2$ centers in III-V compounds or the recently observed negative- $U$ effect of hydrogen in semiconductors ${ }^{1}$ are of great importance for the control of electronic and optical properties of materials. Metastability of these centers has its origin in the atomic configuration of impurities. Recently long living excited electronic states of simple substitutional impurities in Ge and Si have been anticipated from $\mathrm{cw}$ microwave absorption and response measurements. ${ }^{2}$ Here we report the observation of a long lasting storage of carriers, existing for milliseconds, in excited electronic states of shallow impurities in GaP:Te, which is consistently identified as the $1 s(E)$ component of the valley-orbit split shallow donor ground state. Far-infrared (FIR) to infrared up-conversion has also been observed proving a luminescence mechanism of recombination. This upconversion in connection with carrier storage in excited states can be important to realize a FIR semiconductor impurity laser.

Tellurium donors, which have a large central cell shift yielding a binding energy of $90 \mathrm{meV}$, have been ionized by short (40 ns) high-power pulses of FIR radiation with photon energies much less than the binding energy of the impurities. Ionization is accomplished by phonon-assisted tunneling in the electric field of the coherent radiation. ${ }^{3}$ This has been proved by measurements of the photoconductive signal as a function of the wavelength, intensity, and temperature. The ionization probability is independent of the wavelength, increases nonlinearly with the electric field $E$ of the radiation like $\exp \left(E^{2} / E_{c}^{2}\right)$ and the corresponding tunneling time, which determines the characteristic field $E_{c}$, and decreases with rising temperature as $1 / T$. The most important result is the temporal response of the sample. The photoconductive signal pulse shows at first a fast component, which decays with the laser pulse. After the radiation has ceased, however, the signal starts to rise again and finally exponentially drops with a strongly temperature-dependent time constant.

The measurements have been carried out on samples of GaP highly doped with Te with a density of 3 and $7 \times 10^{17}$ $\mathrm{cm}^{-3}$. The resistance of the samples depends on the tem- perature as $\exp \left(-\Delta \varepsilon / k_{B} T\right)$ with activation energy $\Delta \varepsilon$ about half of the binding energy, which shows that the compensation in the material is very low. The density of ionized impurities, $\mathrm{Te}^{+}$, can be estimated to be less than $5 \times 10^{16}$ $\mathrm{cm}^{-3}$ at $T$ below $150 \mathrm{~K}$. The samples were placed in a temperature-variable optical cryostat. Measurements have been carried out in the temperature range between 20 and $150 \mathrm{~K}$ where in thermal equilibrium most of the charge carriers are bound to the impurity ground states. The radiation source was a high-power pulsed far-infrared molecular laser optically pumped by a TEA $\mathrm{CO}_{2}$ laser. Using $\mathrm{NH}_{3}$ as active laser gas, 40-ns pulses with a peak power of $50 \mathrm{~kW}$ have been obtained at wavelengths $\lambda$ of $76,90.5,148$, and 280 $\mu \mathrm{m}$. The photon energies corresponding to these wavelengths are $16.3,13.7,8.3$, and $4.4 \mathrm{meV}$, respectively. In all cases the energy of the quanta is substantially smaller than the ionization energies of the impurities. A series of cold and warm black polyethylene (1 mm thick), teflon and crystal quartz windows were used to transmit far-infrared radiation while rejecting near-infrared and visible light. The ionization of impurities due to high-power pulsed far-infrared radiation has been measured by the usual method of detecting photoconductivity. The photoconductivity measurements have been carried out using a standard $50-\Omega$ load resistor circuit, taking care that the bias voltage across the sample was substantially below the threshold of electric breakdown being about $5 \mathrm{~V} / \mathrm{cm}$. The experimental setup is shown schematically in the inset of Fig. 2.

The irradiation of the samples with far-infrared pulses leads to an increase of the conductivity of the samples. The density of photoexcited carriers can be as large as $10^{14}$ $\mathrm{cm}^{-3}$, which is more than free-carrier concentration in the dark. In Figs. 1 and 2 typical pulse shapes are shown for different time scales. Figure 1 shows the response of a sample on a short time scale and a laser pulse recorded with a linear photon drag detector. The fast signal pulse during irradiation is truncated to show more clearly the strange behavior of the response. During the irradiation the response rises and falls following the excitation laser pulse, being 


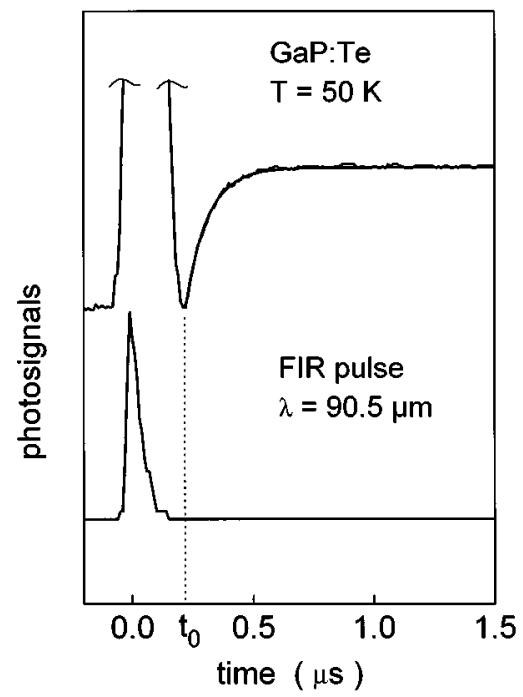

FIG. 1. Upper curve: Photoconductive response of Te-doped $\mathrm{GaP}$ at $T=50 \mathrm{~K}$ on a short (40 ns) single laser pulse of $\lambda=90.5$ $\mu \mathrm{m}$ on a short time scale. The smooth line for $t>t_{0}$ is calculated after Eq. (6). Lower curve: Exciting laser pulse detected by a linear detector.

characterized by times less than 10 ns. However, after the radiation ceased, the signal starts to increase again, assumes a maximum after about $1 \mu \mathrm{s}$, and then decreases exponentially (Fig. 2). The rise after irradiation can very well be fitted by the function $a\left\{1-\exp \left[\left(t-t_{0}\right) / \tau_{r}\right]\right\}$ with a characteristic time $\tau_{r}$ of about $10^{-7} \mathrm{~s}$. The characteristic time $\tau$ of the slow exponential decay (Fig. 2) increases by almost three orders of magnitude with falling temperature from $150 \mathrm{~K}(6$ $\mu \mathrm{s})$ to $35 \mathrm{~K}(3 \mathrm{~ms})$. Figure 3 shows $1 / \tau$ as a function of the inverse temperature. This strong temperature dependence can be fitted in a first approximation by the exponential function $1 / \tau=1 / \tau_{0}[\exp (-\Delta \varepsilon / k T]$ with $\Delta \varepsilon=28 \mathrm{meV}$. The decay time $\tau$ is independent of intensity and of frequency of the radiation.

In addition, up-conversion of FIR radiation into a mid-IR radiation has been observed. A sample of bulk GaP:Te has been placed in front of a fast $\mathrm{Ge}: \mathrm{Cu}$ extrinsic infrared detec-

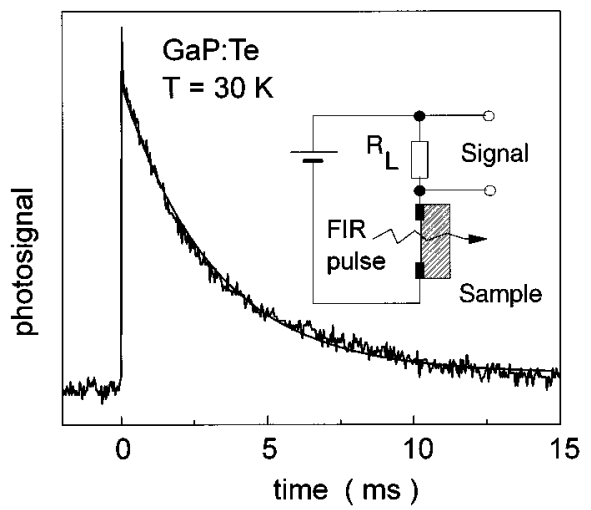

FIG. 2. Photoconductive response of Te-doped GaP at $T=30 \mathrm{~K}$ on a short (40 ns) single laser pulse of $\lambda=90.5 \mu \mathrm{m}$ on a long time scale. The smooth exponentially decaying curve is calculated after Eq. (6). In the inset the experimental setup is shown schematically.
Temperature (K)

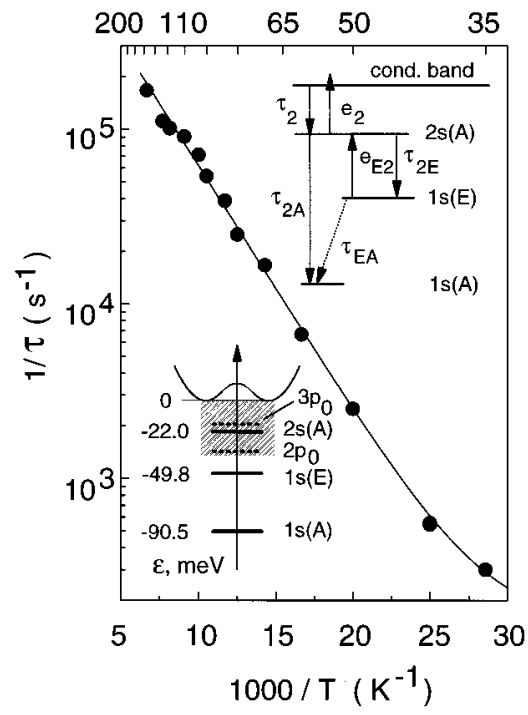

FIG. 3. Temperature dependence of experimentally observed decay time (circles) and fitting curve (line) $1 / \tau$ $=\left[1.6 \times 10^{6} \exp \left(-\Delta \varepsilon_{2 E} / k T\right)+1.44 \times 10^{2}\right] \mathrm{s}^{-1}$. Insets show the structure of Coulombic states of $\mathrm{Te}$ in $\mathrm{GaP}$ (bottom left) and a schematic representation of the kinetic model (top right).

tor, which is sensitive to radiation of wavelengths less than $30 \mu \mathrm{m}(\hbar \omega>40 \mathrm{meV})$. A 2-mm-thick KRS-5 slab has been used as a cutoff filter, which is transparent for wavelengths less than $50 \mu \mathrm{m}$ and blocks totally the exciting laser beam (see inset of Fig. 4). Irradiation of the GaP:Te sample by high-power FIR radiation in the wavelength range of 76-280 $\mu \mathrm{m}$ generates a luminescence signal, which has been detected in the temperature range between 20 and $100 \mathrm{~K}$. The observed luminescence signal is shown in Fig. 4 in comparison to the exciting FIR laser pulse. Without the sample no signal was detected.

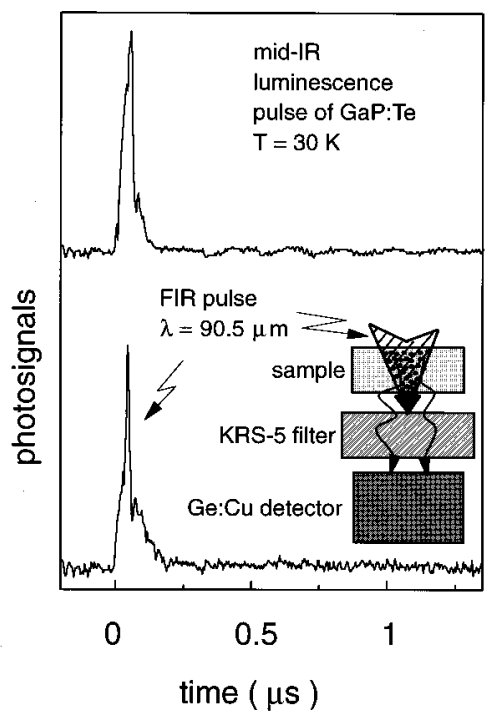

FIG. 4. Mid-IR luminescence signal of Te-doped GaP at $T=40$ $\mathrm{K}$ (upper curve) in response to a short (40 ns) far-infrared laser pulse of $\lambda=90.5 \mu \mathrm{m}$ (lower curve). The inset shows a sketch of the experimental setup. 
Heating of the free-electron gas can be ruled out as a dominant mechanism of the photoconductive signal. Above about $70 \mathrm{~K}$ the mobility of electrons in GaP decreases with rising temperature. ${ }^{4,5}$ Therefore a decrease of conductivity would be expected upon electron heating in contrast to the observation. Furthermore, heating of the sample as a whole cannot lead to the particular time dependence of the slow signal. Finally it is hard to imagine that a five times change in temperature that changes the time constant of the exponential decay by three orders of magnitude may be attributed to a cooling of the sample.

The fast component of the signal is simply due to ionization and rapid capture in excited Coulombic states of Te. ${ }^{6}$ The problem is the rise and decay of the slow component.

Additional deep impurities such as oxygen, which has a very small capture cross section of $10^{-22} \mathrm{~cm}^{2}, 7,8$ can be excluded. They could be responsible for the slow decay but not for the rise of the signal. Obtaining the observed long decay time needs an oxygen density of $5 \times 10^{17} \mathrm{~cm}^{-3}$, which contradicts the low compensation of the investigated material. Additionally only deep centers ionized by the radiation can contribute to capture. This leads to an intensitydependent capture time that has not been observed.

The large capture cross section of Coulombic centers is caused by a rapid capture of free carriers in highly excited states cascading down to the ground state. The last step requires an essentially longer time because of the large energy separation between excited states and the ground state. In spite of the fact that Te is a shallow donor it has a relatively big binding energy for the ground state. For deep centers this last step is governed by multiphonon processes or optical transitions. In the framework of this model the stationary lifetime of excess carriers is controlled by the rapid capture in excited states. The dynamic time in this case consists of two components, a fast one and a slow one. The slow decay time, however, is either independent of temperature or increases with rising temperature in contrast to the present measurements. ${ }^{9}$ Without further modification such a cascade capture model cannot give a rise of the signal after irradiation and the observed temperature dependence of the slow decay. It will be shown, however, that the introduction of an additional long living state, which has vanishingly small recombination probability to the ground state, yields the whole kinetic of the response. This state stores carriers and reinjects them by thermal activation into the densely lying Coulombic states close to the band edge. By this the stored carriers are slowly introduced in the fast recombination channel. The nonequilibrium population distribution of carriers is shifted up in energy, increasing the concentration in the band.

The inset (bottom left) of Fig. 3 shows the structure of the camel's back bottom of the conduction band and the levels of the ground and lowest excited states of Te based on results of Ref. 10. In GaP the valley-orbit splitting causes a large separation of $40.7 \mathrm{meV}$ between states $1 s(E)$ and $1 s(A)$. Cascade capture goes predominantly across $s$ states ${ }^{9}$ by emission of acoustic phonons. Electrons are accumulated in the lowest excited $s$ state $1 s(E)$ because the energy separation between that state and the ground state is much bigger than the largest acoustic phonon energy [31.5 meV, Ref. $10)]$, but still smaller than optical phonon energy [51 meV (Ref. 10)]. The most probable recombination of the electron in the $1 s(E)$ state is by one-phonon excitation to the next higher $s$ state $2 s(A)$, which lies $28 \mathrm{meV}$ above the $1 s(E)$ state. The energy $28 \mathrm{meV}$, however, is just the energy that fits the experimentally determined exponential temperature dependence of the slow decay time $\tau$. Thus, the $1 s(E)$ state may be identified as the level of electron storage. The $2 s(A)$ state is close to several $p$ states therefore, by the exchange between these states due to absorption and emission of acoustical phonons, radiative recombination to the ground state is possible. This explains the observed infrared luminescence.

Based on this assumption a kinetic model is introduced, which is sketched as an inset (top right) of Fig. 3. In agreement with the experimental conditions at low temperatures and the high excitation level, it is assumed that the excess population of excited states is negligible compared to the thermal population in the dark. After irradiation, when there is no more generation, the corresponding rate equations are given by

$$
\begin{gathered}
\frac{d n}{d t}=-\frac{n}{\tau_{2}}+e_{2} n_{2}, \\
\frac{d n_{2}}{d t}=\frac{n}{\tau_{2}}-e_{2} n_{2}-\frac{n_{2}}{\tau_{2 E}}+e_{E 2} n_{E}-\frac{n_{2}}{\tau_{2 A}} \\
\frac{d n_{E}}{d t}=\frac{n_{2}}{\tau_{2 E}}-e_{E 2} n_{E}-\frac{n_{E}}{\tau_{E A}},
\end{gathered}
$$

where $n, n_{2}$, and $n_{E}$ are the densities of electrons in conduction band, the $2 s(A)$ and $1 s(E)$ states, respectively. The time constants $\tau_{2}, \tau_{2 E}, \tau_{2 A}$, and $\tau_{E A}$ and the probabilities of reverse processes $e_{2}$ and $e_{E 2}$ are indicated in the schematic energy-level scheme Fig. 3 (top right). Thermal emission from the ground state is neglected. The thermal transition probability $e_{E 2}$ being important for the model is given by

$$
e_{E 2}=\frac{1}{\tau_{2 E}} \exp \left(-\Delta \varepsilon_{2 E} / k_{B} T\right),
$$

where $\Delta \varepsilon_{2 E}$ is the energy distance between levels $1 s(E)$ and $2 s(A)$.

The rate equations are solved for times $t>t_{0}$ where $t_{0} \approx$ $100 \mathrm{~ns}$ is the time the radiation ceased. This time is larger than $\tau_{2}$ and, thus, for $t>t_{0}$ a quasiequilibrium between free carriers and the population of $2 s(A)$ is established. In this case the solution of the first rate equation in Eq. (1) is given by $n=e_{2} \tau_{2} n_{2}$, showing that $n_{2}$ determines the density $n$ of nonequilibrium free carriers in the conduction band and thus the photoconductive signal. Introducing $n=e_{2} \tau_{2} n_{2}$ for $t>t_{0}$ only the last two of the rate equations in Eq. (1) must be taken into account. Then the concentration of electrons in the state $2 s(A)$ as a function of time is calculated to be 


$$
\begin{aligned}
n_{2}(t)= & \frac{1}{\left(1 / \tau_{d 1}-1 / \tau_{d 2}\right)}\left\{\left[n _ { 2 } ^ { ( 0 ) } \left(\frac{1}{\tau_{2 A}}+\frac{1}{\tau_{2 E}}-\frac{1}{\tau_{E A}}\right.\right.\right. \\
& \left.\left.-e_{2} \frac{\tau_{2 E}}{\left(\tau_{2 A}+\tau_{2 E}\right)}\right)-e_{E 2} n_{E}^{(0)}\right] \exp \left(-\frac{\left(t-t_{0}\right)}{\tau_{d 1}}\right) \\
& \left.+\left[e_{E 2} n_{2}^{(0)} \frac{\tau_{2 A}}{\left(\tau_{2 A}+\tau_{2 E}\right)}+e_{E 2} n_{E}^{(0)}\right] \exp \left(-\frac{\left(t-t_{0}\right)}{\tau_{d 2}}\right)\right\},
\end{aligned}
$$

where $n_{2}^{(0)}$ and $n_{E}^{(0)}$ are the concentrations of the $2 s(A)$ and $1 s(E)$ states at $t=t_{0}$, respectively. The dynamic relaxation times $\tau_{d 1}$ and $\tau_{d 2}$ are given by

$$
\begin{gathered}
\frac{1}{\tau_{d 1}}=\frac{1}{\tau_{2 A}}+\frac{1}{\tau_{2 E}}+e_{E 2} \frac{\tau_{2 A}}{\left(\tau_{2 A}+\tau_{2 E}\right)}, \\
\frac{1}{\tau_{d 2}}=e_{E 2} \frac{\tau_{2 E}}{\left(\tau_{2 A}+\tau_{2 E}\right)}+\frac{1}{\tau_{E A}} .
\end{gathered}
$$

The assumption that carriers are dominantly captured by the $1 s(E)$ state, i.e., $\tau_{2 E} \ll \tau_{2 A}$, leads to $n_{E}^{(0)} \gg n_{2}^{(0)}$ Thus, in Eq. (3) terms proportional to $n_{2}^{(0)}$ can be neglected in comparison to those proportional to $n_{E}^{(0)}$ yielding

$$
\begin{aligned}
n_{2}(t) \approx & \frac{e_{E 2} n_{E}^{(0)}}{\left(1 / \tau_{d 1}-1 / \tau_{d 2}\right)}\left[\exp \left(-\frac{\left(t-t_{0}\right)}{\tau_{d 2}}\right)\right. \\
& \left.-\exp \left(-\frac{\left(t-t_{0}\right)}{\tau_{d 1}}\right)\right]
\end{aligned}
$$

As it is seen, the rise and the following exponential decay depending on the temperature follows from Eq. (6) if $\tau_{d 1}<\tau_{d 2}$. Fitting of Eq. (6) with $\tau_{d 1}$ and $\tau_{d 2}$ as free parameters to the experimentally observed rise and decay of the slow signal is shown in Figs. 1 and 2. The resulting dynamic time constants are $1 / \tau_{d 1}=10^{7} \mathrm{~s}^{-1}$ and $1 / \tau_{d 2}$ $=1.6 \times 10^{6} \exp \left(-\Delta \varepsilon_{2 E} / k T\right)+1.44 \times 10^{2}$ with $\left(\Delta \varepsilon_{2 E}=28\right.$ $\mathrm{meV})$. The temperature dependence of $\tau_{d 2}$ after the latter relation is shown in Fig. 3 by the full line. The two dynamic relaxation times differ at least by two orders of magnitude and, in fact, a one-parameter fitting procedure was sufficient for the whole temperature range. All numeric results are in excellent agreement with the measurements.

It can be shown that for the present temperature range the last term in Eq. (4) is much smaller than $1 / \tau_{2 A}+1 / \tau_{2 E}$. Omitting this term allows one to determine all three kinetic time constants from the measured dynamic times and the temperature dependence of $\tau_{d 2}$. We find $\tau_{2 E}=10^{-7} \mathrm{~s}, \tau_{2 A}$ $=5 \times 10^{-7} \mathrm{~s}$, and $\tau_{E A}=0.7 \times 10^{-2} \mathrm{~s}$. This result is consistent with the assumptions of the model that $\tau_{2 E} \ll \tau_{2 A}$ $\ll \tau_{E A}$. Finally we note that Eq. (6) yields a maximum of $n_{2}$ and the corresponding photoconductive signal which occurs at the time $t_{1}=\tau_{d 1}\left(\Delta \varepsilon_{2 E} / k_{B T}\right) \ln \left(\tau_{2 A} / \tau_{2 E}\right)$ and shifts with temperature. This effect has also been observed.

In summary, a storage of electrons in an excited shallow donor state has been observed with a lifetime that can be as long as several milliseconds. This level could be consistently identified as the $1 s(E)$ component of the valley-orbit split shallow donor ground state. The experimental observations have been described by a cascade capture model. Carriers rapidly captured within 100 ns by highly excited Coulomb states are accumulated in the $1 s(E)$ state, which has a very longlife time of about $10 \mathrm{~ms}$ for direct transitions into the ground state. The recombination of carriers from this state takes place by thermal excitation into somewhat higher-lying $s$ and $p$ states and subsequent optical and, probably, acoustic multiphonon transitions. Optical transitions have been detected by the observation of mid-infrared luminescence. The high excess carrier density in the excited impurity state has been observed up to a temperature of $150 \mathrm{~K}$ and may be of interest for the development of a semiconductor impurity laser in the infrared. Population inversion of radiative states could be realized by reexciting the carriers from the storage level applying an additional far-infrared pulse. However, the use of far-infrared laser pulses is not stringent. Indeed, no specific far-infrared features, except the high electric field strength of the laser pulse, have been utilized to excite free carriers. The generation of free carriers and the re-injection of stored carriers in the radiative recombination channel could also be accomplished by short electric dc pulses.

Financial support by the Deutsche Forschungsgemeinschaft is gratefully acknowledged. The authors thank W. Huber for providing software used in the work.
* On leave from A.F. Ioffe Physicotechnical Institute of the Russian Academy of the Sciences, St. Petersburg, 194021, Russia.

${ }^{1}$ J. Neugebauer and C. G. Van de Walle, Phys. Rev. Lett. 75, 4452 (1995).

${ }^{2}$ Ya.E. Pokrovskii, O.I. Smirnova, and N.A. Khvalkovskii, Solid State Commun 93, 405 (1995)

${ }^{3}$ S. D. Ganichev, W. Prettl, and P. G. Huggard, Phys. Rev. Lett. 71, 3882 (1993).

${ }^{4}$ D.N. Nasledov, V.V. Negreskul, S.I. Radautsan, S.V. Slobodchikov, Phys. Status Solidi 10, 37 (1965).

${ }^{5}$ H.C. Montgomery, J. Appl. Phys. 39, 2002 (1968).
${ }^{6}$ R.Z. Bachrach, P.D. Darkus, and O.G. Lorimor, J. Appl. Phys. 45, 4971 (1974).

${ }^{7}$ C.H. Henry and D.V. Lang, Phys. Rev. B 15, 989 (1977).

${ }^{8}$ P. Dean, Oxygen in Gallium Phosphide, Deep Centers in Semiconductors (Gordon and Breach Science Publishers, New York, 1986).

${ }^{9}$ V.N. Abakumov, V.I. Perel, and I.N. Yassievich, Nonradiative Recombination in Semiconductors, Modern Problems in Condensed Matter Sciences Vol. 33 (North-Holland, Amsterdam, 1991).

${ }^{10}$ W. Scott, J. Appl. Phys. 50, 472 (1979). 